\title{
Arrest at the diplotene stage of meiotic prophase I is delayed by progesterone but is not required for primordial follicle formation in mice
}

\author{
Sudipta Dutta ${ }^{1,2}$, Deion M. Burks ${ }^{1}$ and Melissa E. Pepling ${ }^{1 *}$
}

\begin{abstract}
Background: In mammalian females, reproductive capacity is determined by the size of the primordial follicle pool. During embryogenesis, oogonia divide mitotically but cytokinesis is incomplete so oogonia remain connected in germ cell cysts. Oogonia begin to enter meiosis at 13.5 days postcoitum in the mouse and over several days, oocytes progress through the stages of meiotic prophase I arresting in the diplotene stage. Concurrently, germ cell cysts break apart and individual oocytes become surrounded by granulosa cells forming primordial follicles. In rats, inhibition of a synaptonemal complex protein caused premature arrival at the diplotene stage and premature primordial follicle assembly suggesting diplotene arrest might trigger primordial follicle formation. Cyst breakdown and primordial follicle formation are blocked by exposure to steroid hormones but hormone effects on the timing of diplotene arrest are unclear. Here, we asked: (1) if oocytes were required to arrest in diplotene before follicles formed, (2) if all oocytes within a germ cell cyst arrested at diplotene synchronously, and (3) if steroid hormones affected progression through prophase I.
\end{abstract}

Methods: Meiotic stage and follicle formation were assessed in histological sections. Statistical differences over time were determined using one-way ANOVA followed by Newman-Keuls multiple comparisons test. To determine if steroid hormones affect the rate of progression to the diplotene stage, $17.5 \mathrm{dpc}$ ovaries were placed in organ culture with media containing estradiol, progesterone or both hormones. In this case, differences were determined using one-way ANOVA followed by Dunnett's multiple comparisons test.

Results: We found primordial follicles containing oocytes at the diplotene stage as well as follicles containing oocytes at pre-diplotene stages. We also found individual germ cell cysts containing oocytes at both diplotene and pre-diplotene stages. Progesterone but not estradiol reduced the number of diplotene oocytes in ovary organ culture.

Conclusions: Our results suggest that meiotic progression and primordial follicle formation are independent events. In addition, oocytes in germ cell cysts do not synchronously proceed through meiosis. Finally, only progesterone delayed transit though meiotic prophase I.

Keywords: Fetal oocyte development, Steroid hormones, Diplotene arrest, Meiotic prophase I progression, Primordial follicle formation

\footnotetext{
* Correspondence: mepeplin@syr.edu

'Department of Biology, Syracuse University, 107 College Place, Syracuse, NY

13244, USA

Full list of author information is available at the end of the article
} 


\section{Background}

In female mammals, reproductive capacity is determined at birth by the non-renewable pool of primordial follicles present, representing the total population of germ cells available for reproductive purposes [1]. In mice, the primordial germ cells migrate to the genital ridge and divide by mitosis until 13.5 days postcoitum (dpc) [2]. During these divisions the germ cells are known as oogonia and develop in germ cell cysts due to incomplete cytokinesis following each cell cycle [3]. Oogonia start to enter meiosis at approximately $13.5 \mathrm{dpc}$ and are then referred to as oocytes [2]. Oogonia do not appear to enter meiosis synchronously $[2,4]$. However, meiosis proceeds from anterior to posterior suggesting that local factors diffuse from the mesonephros at the anterior side of the ovary to promote meiosis [5]. Most germ cells have entered meiosis by 15.5 dpc [2]. After entering meiosis oocytes progress through the initial stages of meiotic prophase I and remain arrested at the diplotene stage until just prior to ovulation. Some oocytes arrive at the diplotene stage by $17.5 \mathrm{dpc}$ but it takes several days until all oocytes are in diplotene [2]. During the same time period, germ cell cysts break apart and individual oocytes become surrounded by granulosa cells forming primordial follicles [6]. Some follicles, referred to as the first wave of developing follicles are activated to grow immediately after forming while most follicles are not activated until sexual maturity developing in groups in a cyclical fashion [7, 8].

Mutations in genes responsible for the initial stages of meiosis in mouse such as disrupted meiotic cDNA 1 (Dmc1) and meiosis-specific sporulation protein (Spo11) result in loss of oocytes and an inability to form follicles which ultimately cause sterility $[9,10]$. Meiotic prophase I is marked by the expression of synaptonemal complex proteins (SYCPs) that make up the synaptonemal complex which is required for DNA synapsis and meiotic recombination between homologous chromosomes. In rats, inhibition of SYCP1 accelerated arrival at the diplotene stage along with premature assembly of those oocytes into primordial follicles [11]. In fetal bovine ovaries, many oocytes do not appear to arrest at diplotene but instead continue on through diakinesis where they are eventually lost by attrition [12]. A crucial factor for the survival of germ cells may be their ability to be enclosed within follicles [13]. This evidence reflects the possibility of a link between two events that occur during early mouse oogenesis, arrest at the diplotene stage and primordial follicle formation.

Both estradiol $\left(\mathrm{E}_{2}\right)$ and progesterone $\left(\mathrm{P}_{4}\right)$ can delay cyst breakdown and primordial follicle formation [14]. Estrogens have also been shown to affect meiotic progression of oocytes. When pregnant mice were treated with bisphenol A (BPA), a known estrogenic compound, oocytes from female fetuses of exposed mothers had synaptic defects and recombination aberrations [15]. In adults, those aberrations gave rise to aneuploid eggs and embryos. In cattle, it is thought that primordial follicles cannot be activated until after the oocyte reaches diplotene arrest. In vitro treatment of bovine ovaries with estradiol $\left(\mathrm{E}_{2}\right)$ or progesterone $\left(\mathrm{P}_{4}\right)$ decreased the number of follicles that were activated and thus, may affect progression to the diplotene stage [16].

The main objectives of the present study were to determine if diplotene arrest is linked with primordial follicle formation in mice and to elucidate the role of steroid hormone signaling in meiotic progression of murine oocytes. The molecular mechanisms involved in regulating progression through prophase I and in primordial follicle formation in the developing ovary are still poorly understood. Elucidating events during fetal ovarian development will increase our understanding of the factors that control oocyte quality and quantity and thus help in improvement of current infertility treatments.

\section{Methods \\ Animals}

Adult CD1 male and female outbred mice were obtained from Charles River Laboratories (Wilmington, MA, USA) and were maintained in accordance with the policies of Syracuse University's Institutional Animal Care and Use Committee. Mice were housed and bred at a controlled photoperiod (14 h light, $10 \mathrm{~h}$ dark), temperature $\left(21-22{ }^{\circ} \mathrm{C}\right)$, and humidity with food and water available ad libitum. CD1 females were mated with males of the same strain and checked daily for vaginal plugs. Noon on the day of vaginal plug detection was designated as $0.5 \mathrm{dpc}$. Birth usually occurred at $19.5 \mathrm{dpc}$ and was designated as postnatal day (PND) 1. Pregnant mice were euthanized by $\mathrm{CO}_{2}$ asphyxiation for fetal ovary collection. For neonatal ovary collection, pups were euthanized by decapitation on the appropriate day.

\section{Histological methods}

Fetal and neonatal ovaries were dissected and trimmed of extra tissue. They were fixed in Bouin's solution for $2 \mathrm{~h}$ at room temperature, followed by dehydration through an ethanol series. Histological processing of the ovaries was performed at the Electron Microscopy \& Histology Core Facility, Weill Cornell Medical College, New York. Following standard protocols for paraffin-embedded sections, ovaries were serially sectioned at $5 \mu \mathrm{m}$ and stained with hematoxylin and eosin. Images were taken on an Olympus BX50 microscope with an Olympus DP71 digital camera. Every fifth section was marked for direct counts of oocytes at pre-diplotene and diplotene stages and four to five ovaries were analyzed at each age. A total of 10-12 sections per ovary were used for counting. To avoid bias, all ovaries were analyzed without knowledge of age. To avoid double counting of oocytes, only oocytes having a visible nucleus were counted. Oocytes at pre-diplotene stages 
were characterized at the leptotene stage by the appearance of thin long threads of chromatin, at the zygotene stage by regions of thicker chromosome strands and at the pachytene stage by thick highly condensed chromatin. Oocytes at the diplotene stage were recognized by areas of condensed chromatin interspersed with clear areas [17]. The total number of pre-diplotene and diplotene oocytes for each ovary was determined by multiplying the number of pre-diplotene and diplotene oocytes by five to account for every fifth section being used in the analysis. Oocytes were counted as cysts if they were present in clusters of at least two oocytes without any intervening somatic cells. Oocytes were considered to be in primordial follicles if they contained an oocyte surrounded by a layer of flattened granulosa cells.

\section{In vitro ovary organ culture}

Ovaries dissected at $17.5 \mathrm{dpc}$ were placed in culture. Ovaries were cultured in 4-well culture plates in drops of media on $0.4 \mu \mathrm{M}$ floating filters (Millicell- CM; Millipore Corp., Bedford, MA) in 0.4 ml DMEM-Ham's F-12 media supplemented with penicillin-streptomycin, 5X ITS-X (Life Technologies, Inc., Grand Island, NY), 0.1\% BSA, 0.1\% albumax, and $0.05 \mathrm{mg} / \mathrm{ml} \mathrm{L}$-ascorbic acid. $\mathrm{E}_{2}$ and $\mathrm{P}_{4}$ (Sigma Chemical Co., St. Louis, MO) were dissolved in dimethylsulfoxide (DMSO) at a concentration of $0.1 \mathrm{M}$ and then added to culture media to achieve the desired final concentration. DMSO was added to media at the same percent as the chemical to serve as vehicle control. Ovaries were placed in culture and exposed daily to $\mathrm{E}_{2}, \mathrm{P}_{4}$ or both hormones at 10

${ }^{-6} \mathrm{M}$ or DMSO alone ( $n=5$ ovaries per treatment group). Ovaries were divided randomly among the treatment groups. The ovaries from control and treatment were fixed in Bouin's fixative and histologically processed.

\section{Statistical analysis}

The percent of oocytes in diplotene, percent of oocytes in follicles and percent of follicles containing pre-diplotene oocytes over time were calculated using four to five ovaries at each time point. Data are represented as mean \pm SEM of nontransformed data. As counted data by nature are nonnormally distributed, logarithmic transformation $(\mathrm{Y}=\log$ [y]) was performed on the data. Statistical analyses using transformed data were performed using GraphPad Prism version 6 (GraphPad Software, San Diego, CA). Statistical differences $(P<0.05)$ among the means were evaluated using one-way ANOVA followed by Newman-Keuls multiple comparisons test. Effects of $\mathrm{E}_{2}$ and $\mathrm{P}_{4}$ on oocyte number, percent single oocytes, percent oocytes in diplotene and percent of follicles containing diplotene oocytes were analyzed using one-way ANOVA followed by Dunnett's multiple comparisons test using five ovaries per treatment group. All results are presented as mean \pm SEM of nontransformed data.

\section{Results \\ Meiotic progression and diplotene arrest in developing mouse ovaries}

To investigate the relationship between meiotic progression and primordial follicle formation, we first performed a quantitative study on histological sections from $13.5 \mathrm{dpc}$ to PND5, determining the number of oocytes at each meiotic stage. The first diplotene oocytes were observed at 17.5 dpc, when about $8 \%$ of the oocytes were at the diplotene stage (Fig. 1a). A statistically significant increase to $32 \%$ diplotene stage oocytes was observed at PND1, to $49 \%$ at PND2 and to $86 \%$ at PND3. A slight but non-significant increase was observed in PND5 ovaries.

Representative histological sections with examples of the different meiotic prophase I stages are shown in Fig. 1b-1e. Figure $1 \mathrm{~b}$ shows a section from a $14.5 \mathrm{dpc}$ ovary with oocytes at the leptotene stage identified by long thin nuclear threads as the chromatin begins to condense $[16,17]$. Figure 1c shows a section from a $15.5 \mathrm{dpc}$ ovary with oocytes at the zygotene stage characterized by regions of thicker chromatin as homologous chromosomes begin to pair. Figure 1d shows a 17.5 dpc ovary with oocytes at the pachytene stage distinguished by very thick strands of chromatin. Figure 1e shows a PND5 ovary with oocytes at diplotene characterized by areas of condensed chromatin separated by clear areas [17].

\section{Primordial follicle formation and diplotene arrest are independent processes}

Previous studies from our lab have indicated that in the medullary region, primordial follicles are found as early as 17.5 $\mathrm{dpc}$ [6]. Here we performed a thorough investigation analyzing serial sections $5 \mu \mathrm{m}$ apart covering the entire ovary thereby enabling us to obtain a more accurate estimate regarding the presence of primordial follicles. The earliest primordial follicles were detected at $16.5 \mathrm{dpc}$ where we found $2 \%$ of the oocytes enclosed in follicles (Fig. 2a). We hypothesized that oocytes needed to reach diplotene before becoming enclosed in follicles. However, we observed prediplotene oocytes both in germ cells cysts (Fig. 2c) and in primordial follicles (Fig. 2d). Figure $2 \mathrm{~b}$ shows the percentage of follicles containing pre-diplotene oocytes from $15.5 \mathrm{dpc}$ to PND5. All of the follicles observed at $16.5 \mathrm{dpc}$ were at the pre-diplotene stage. For all later stages, only a small percentage of follicles contained pre-diplotene oocytes with the majority already at diplotene (Fig. 2f). We also observed oocytes still in germ cell cysts that were at the diplotene stage (Fig. 2e). Thus, meiotic progression and primordial follicle formation do not appear to be linked.

\section{All oocytes in a cyst do not reach the diplotene stage synchronously}

Oocytes enter meiosis in a wave from anterior to posterior in the mouse ovary. However, it is not known within 

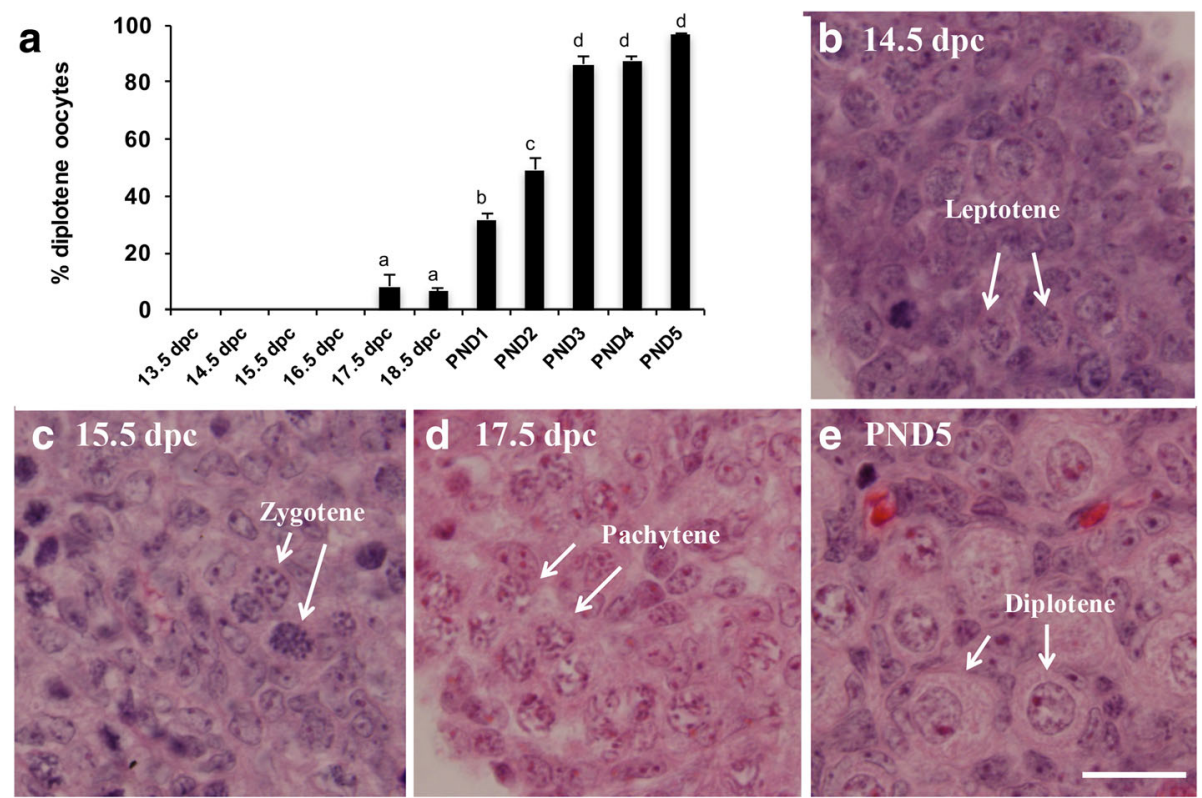

Fig. 1 Diplotene stage oocytes are first detected at $17.5 \mathrm{dpc}$. a Percentage of oocytes at the diplotene stage of meiotic prophase I $( \pm$ SEM) in perinatal mouse ovaries from $13.5 \mathrm{dpc}$ to PND5. Different letters indicate a significant difference between groups $(P<0.05 ; n=4-5$ ovaries per time point) as determined by one-way ANOVA followed by Newman-Keuls multiple comparisons test. b-e Representative histological sections of perinatal mouse ovaries stained with hematoxylin and eosin showing different meiotic prophase I stages indicated by white arrows. b $14.5 \mathrm{dpc}$ ovary showing oocytes at leptotene. c 15.5 dpc ovary showing oocytes at zygotene. d $17.5 \mathrm{dpc}$ ovary showing oocytes at pachytene. e PND5 ovary showing oocytes at diplotene. Scale bar $=20 \mu \mathrm{M}$
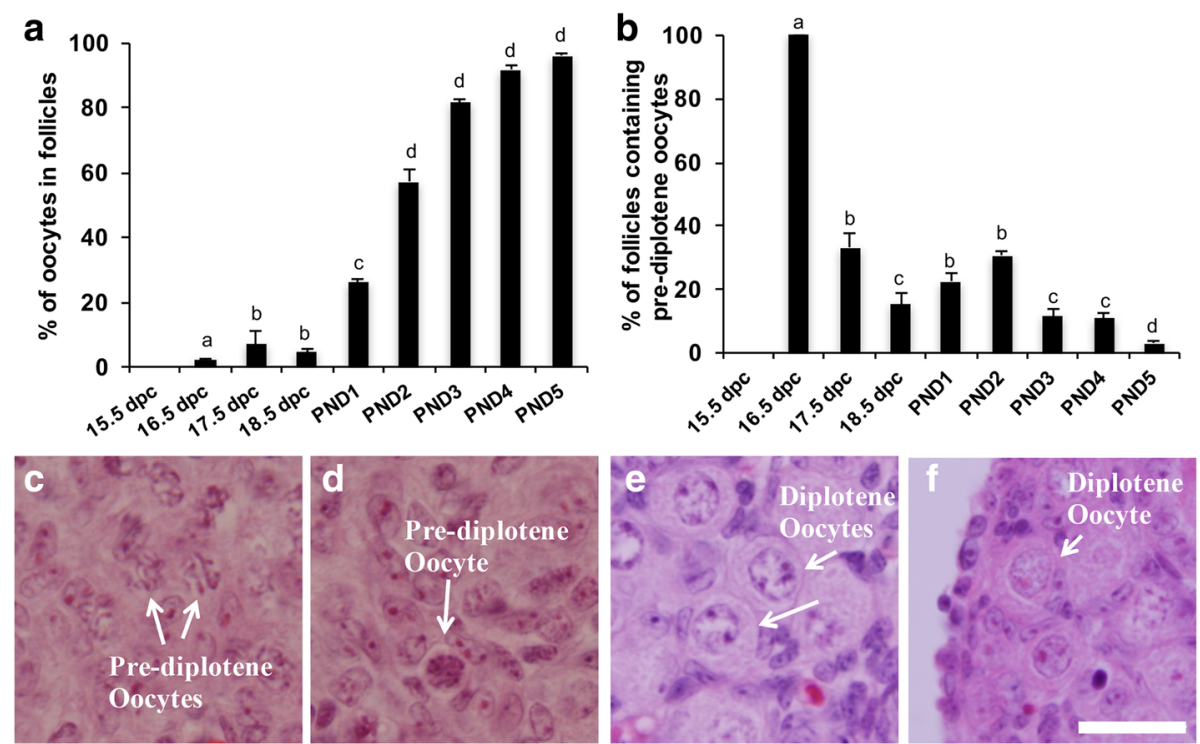

Fig. 2 Primordial follicle formation and meiotic progression in perinatal mouse ovaries. a Percentage of oocytes in follicles ( \pm SEM) over time. Different letters indicate a significant difference between groups $(P<0.05 ; n=4-5$ ovaries per time point) as determined by one-way ANOVA followed by Newman-Keuls multiple comparisons test. $\mathbf{b}$ Percentage of follicles with oocytes at the pre-diplotene stage out of total follicles ( \pm SEM) in perinatal mouse ovaries. Different letters indicate a significant difference between groups $(P<0.05 ; n=4-5$ ovaries per time point) as determined by one-way ANOVA followed by Newman-Keuls multiple comparisons test. c-f Representative histological images of perinatal mouse ovaries stained with hematoxylin and eosin. c 17.5 dpc ovary showing pre-diplotene oocytes in germ cell cysts indicated by white arrows. d 17.5 dpc ovary showing a primordial follicle containing a pre-diplotene oocyte indicated by a white arrow. e PND3 ovary showing diplotene oocytes not yet enclosed in primordial follicles indicated by white arrows. f PND5 ovary showing a diplotene oocyte in a primordial follicle indicate by a white arrow. Scale bar $=20 \mu \mathrm{M}$ 
individual cysts whether oocytes enter or progress through meiosis and arrest at diplotene synchronously. To determine if all oocytes in a germ cell cyst were at the same meiotic stage, a quantitative analysis of meiotic stages of oocytes present within individual cysts was performed at PND1. We chose this age because many pachytene and diplotene stage oocytes are present and most oocytes are still in germ cell cysts. For each cyst we determined whether an oocyte was at the pre-diplotene or diplotene stage of meiosis. We examined sections from five different ovaries and found cysts with only diplotene oocytes, with only pre-diplotene oocytes and with a mixture of prediplotene and diplotene oocytes (Fig. 3). For each cyst that had a mixture of different stage oocytes we determined the percent of oocytes at each stage. Each cyst contained two to five oocytes visible in the section. We found that individual cysts had an average of 63\% diplotene oocytes. It is important to note that only oocytes in individual sections were analyzed and that the cysts likely contained more oocytes not visible in the section. These findings suggest that oocytes within individual cysts do not progress through meiosis synchronously.

\section{Effects of steroid hormones on meiotic progression}

There is some evidence that steroid hormones can cause delays in meiotic progression. We wanted to know if $E_{2}$ or $\mathrm{P}_{4}$ could delay arrival at the diplotene stage in mouse ovaries. To test this, $17.5 \mathrm{dpc}$ fetal ovaries were placed in organ culture for four days with control media or media containing $10^{-6} \mathrm{M} \mathrm{E}_{2}, 10^{-6} \mathrm{M} \mathrm{P}_{4}$ or both $10^{-6} \mathrm{M} \mathrm{E}_{2}$ and $10^{-6} \mathrm{M}$ $\mathrm{P}_{4}$. These hormone doses were chosen based on our previous studies as doses that reduced primordial follicle assembly in organ culture [14]. After culture, serial sections were prepared, stained with hematoxylin and eosin and analyzed. There was no difference in the number of oocytes between control and treated ovaries (Fig. 4a). There was also no difference in the percent of single oocytes, a measure of primordial follicle formation (Fig. 4b). In addition, there was also no statistically significant difference in the percentage of oocytes at the diplotene stage of prophase I (Fig. 4c).
However, when only oocytes already assembled in primordial follicles were examined, there were significantly fewer follicles containing diplotene oocytes in the $\mathrm{P}_{4}$ treated ovaries (Fig. 4d).

\section{Discussion}

Here we investigated the relationship between two critical events in mammalian oogenesis, primordial follicle formation and diplotene arrest. We confirmed that the earliest diplotene oocytes were observed at $17.5 \mathrm{dpc}$ and gradually increased over time. In previous studies from our lab, the earliest age that primordial follicles were reported was $17.5 \mathrm{dpc}[6]$. However, here we observed a small percentage of oocytes in follicles even earlier, at $16.5 \mathrm{dpc}$. We also found primordial follicles containing pre-diplotene oocytes, supporting the idea that oocytes do not need to reach diplotene arrest before they can become enclosed in primordial follicles. Furthermore, oocytes within individual germ cell cysts do not appear to proceed through meiosis synchronously. Finally, progesterone treatment delayed meiotic progression of oocytes in follicles.

Borum in 1961 reported that mouse oocytes begin to reach the diplotene stage at $17.5 \mathrm{dpc}$ in the Street inbred strain of mice [2]. A more recent study using CD1 outbred mice reported that the first diplotene oocytes were observed at $18.5 \mathrm{dpc}$ [18]. However, for that study, the sample size was limited to only 200 oocyte nuclei at each age. We reexamined this question in the same strain $(C D 1)$ and found oocytes in diplotene arrest as early as $17.5 \mathrm{dpc}$ in agreement with the original studies of Borum. Further, we see an increase in the percent of diplotene oocytes from PND1 to PND3 and no further increase to PND5. Previous reports suggest that most oocytes have arrested in the diplotene stage by PND5 and consistent with this in our studies $97 \%$ of the oocytes were in diplotene at PND5.

Inhibition of the synaptonemal complex protein SYCP1 in rats led to premature arrival of oocytes at the diplotene stage and also resulted in accelerated primordial follicle assembly [11]. These observations suggested that diplotene arrest is developmentally linked with follicle formation in
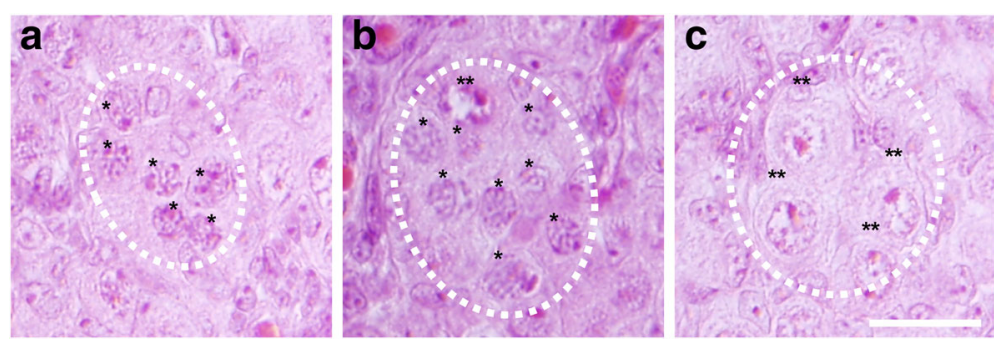

Fig. 3 Representative images showing paraffin sections of PND1 mouse ovaries stained with hematoxylin and eosin. a Ovarian section showing a cyst with all pre-diplotene oocytes circled with a white dashed line. b Ovarian section showing a cyst with both pre-diplotene and diplotene stage oocytes circled by a white dashed line. c Ovarian section showing a cyst with all diplotene oocytes circled with a white dashed line. Pre-diplotene oocytes are indicated by one asterisk and diplotene oocytes are indicated by two asterisks. Scale bar $=20 \mu \mathrm{M}$ 

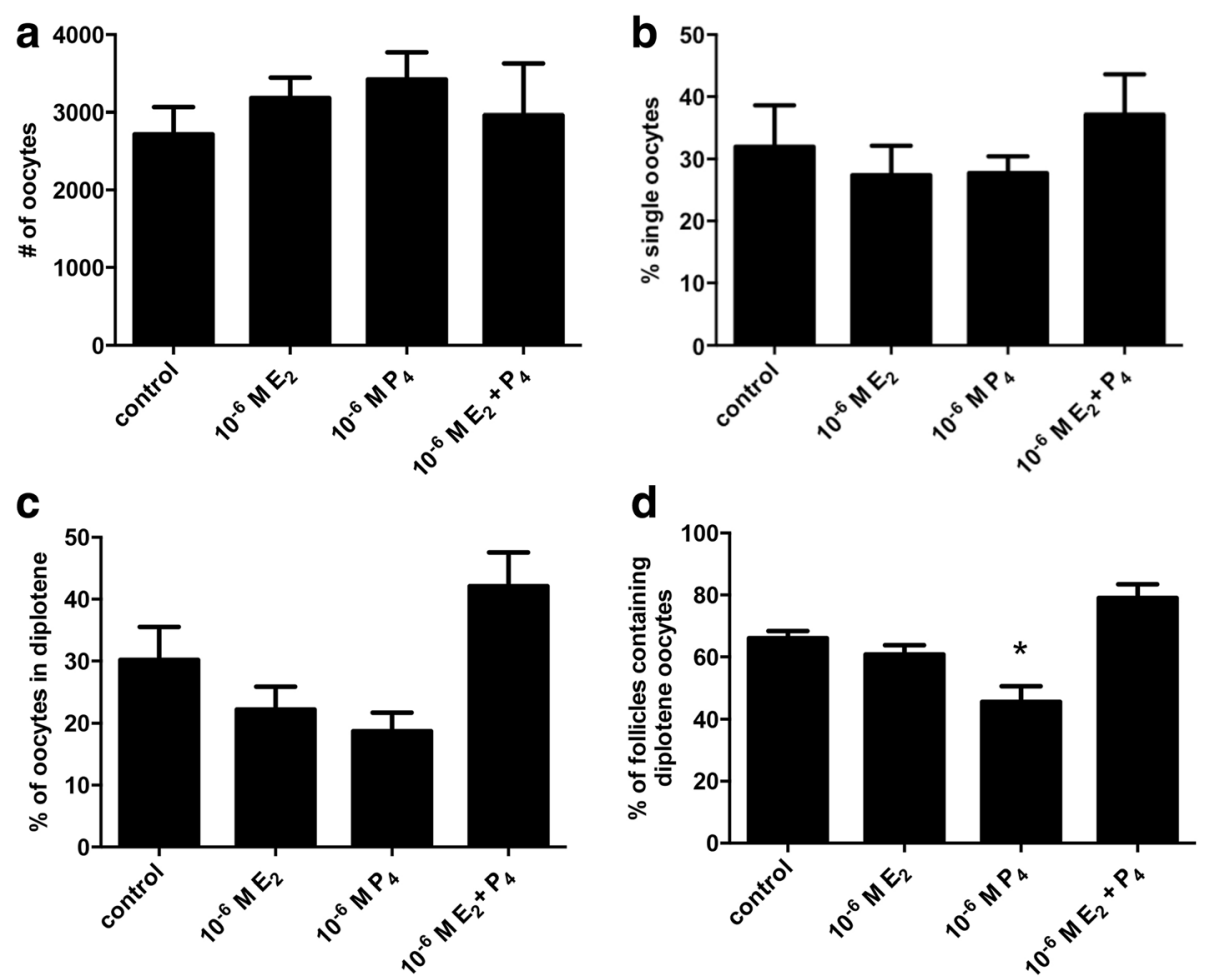

Fig. 4 Effects of $E_{2}$ and $P_{4}$ treatment in organ culture on meiotic stage. a Total number of oocytes, $\mathbf{b}$ percent single oocytes $\mathbf{c}$ percent diplotene oocytes and $\mathbf{d}$ percent follicles containing diplotene stage oocytes per ovary in control ovaries or ovaries treated with $10^{-6} \mathrm{M} \mathrm{E}_{2}, 10^{-6} \mathrm{M} \mathrm{P}_{4}$ or 10

${ }^{-6} \mathrm{ME}_{2}+10^{-6} \mathrm{MP}_{4}$ for 4 days in organ culture. Data are presented as the mean $\pm \mathrm{SEM}$. * indicates a significant difference from control ovaries $(P<0.05 ; n=5$ ovaries per treatment) as determined by one-way ANOVA followed by Dunnett's multiple comparisons test

rodents. Supporting this notion, Wang and colleagues found that in newborn mice, all oocytes in primordial follicles were at the dictyate (late diplotene) stage and no follicles containing earlier stages were observed [19]. However, our results here suggest that meiotic stage and primordial follicle formation are not closely linked. Stra 8 deficient female mice have been previously shown to have a meiotic initiation block at $13.5 \mathrm{dpc}$ to $14.5 \mathrm{dpc}$ [20]. The authors postulated that if oocytes were required to enter meiosis for follicles to assemble then Stra 8 deficient ovarian germ cells would not undergo follicle formation or activation. However, the germ cells in Stra8 deficient ovaries did become enclosed in granulosa cells and developed to advanced stages [21]. Thus, some studies suggest that progression of oocytes to the diplotene stage is a prerequisite to form follicles in rodents while other studies including data presented here do not support this idea. In cattle, up to $80 \%$ of oocytes in primordial follicles are at prediplotene stages during fetal development suggesting that oocytes are not required to reach the diplotene stage before follicles are able to form in bovine ovaries [16]. Further investigation is necessary determine the exact link between primordial follicle formation and meiotic stage.

Here, we examined the effects of $E_{2}$ and $P_{4}$ on progression of oocytes to the diplotene stage. Surprisingly, $\mathrm{P}_{4}$ but not $E_{2}$ delayed meiotic progression, decreasing the percent of follicles containing diplotene oocytes. In previous studies, exposure of pregnant C57BL/6 females to the estrogenic compound BPA, disrupted the process of meiotic prophase [15]. Oocytes from female fetuses had defects in synapsis and increased recombination resulting in higher levels of aneuploidy. This difference may be due to differences in the specific time period examined or route of hormone exposure. BPA exposure spanned $11.5 \mathrm{dpc}$ to $18.5 \mathrm{dpc}$, while the fetal ovaries in our study were harvested at $17.5 \mathrm{dpc}$ and exposed to hormones in organ culture for 4 days. There may also be differences depending on which estrogenic compound is used. Finally, Susiarjo and colleagues examined chromosomal defects while we examined progression to the diplotene stage.

Previous work from our lab demonstrated that exposure to $\mathrm{P}_{4}$ or $\mathrm{E}_{2}$, as well as synthetic estrogens, BPA, diethylstilbestrol or ethinyl estradiol delayed germ cell cyst breakdown and primordial follicle formation [14, 22]. In addition, estrogens at some concentrations also altered the oocyte loss that accompanies cyst breakdown. Here we found that $\mathrm{P}_{4}$ or $\mathrm{E}_{2}$ had no effect on cyst breakdown, primordial follicle formation or oocyte survival. The previous studies began hormone exposure at PND1 while in the current work hormone treatment began earlier at $17.5 \mathrm{dpc}$ and this may be outside the 
window of sensitivity. Interestingly, in a previous study using rats, $\mathrm{P}_{4}$ but not $\mathrm{E}_{2}$ significantly inhibited primordial follicle assembly [23]. In our study neither hormone affected follicle assembly, however, we observed an effect of $\mathrm{P}_{4}$ on meiotic progression. It is also notable that there was no significant effect of $\mathrm{P}_{4}$ and $\mathrm{E}_{2}$ together on meiotic progression. We would expect that since $\mathrm{P}_{4}$ alone affects meiosis, both hormones would also have an effect. $E_{2}$ may activate signals that block the effects of $\mathrm{P}_{4}$.

Here, we examined nuclear morphology using classic histology to analyze meiotic prophase I of perinatal oocytes so that we could address meiotic progression in the context of follicle formation and development. We wanted to use molecular tools such as antibodies that recognize meiotic marker proteins. While there are several such antibodies, most are used in the surface spread technique where oocyte nuclei are spread out and tissue structure is lost so that stage of follicle development for oocytes cannot be determined. Unfortunately, many of the antibodies do not work well in whole mount immunocytochemistry where follicle formation and development could be analyzed. We are in the process of testing meiotic prophase marker antibodies to identify one that does work in whole mount immunocytochemistry to be used in future studies.

\section{Conclusions}

In summary, the processes of primordial follicle formation and meiotic progression to the diplotene stage do not appear to be closely linked in mouse ovaries. $\mathrm{P}_{4}$ reduced the percent of oocytes within primordial follicles that have reached diplotene arrest. In women, diplotene arrested oocytes often remain dormant for many years before being activated. Mechanisms controlling progression of oocytes through prophase I and arrest at the diplotene stage are not well understood but this knowledge will be essential to prevent defects in meiosis such as aneuploidy.

\section{Acknowledgements}

The authors thank Joanne Fortune and Ming Yang for critical reading of the manuscript and Twinkle Chowdhury for advice on statistics. In addition, the authors greatly appreciate the availability of the Pitnick laboratory microscope for analysis of histological sections.

\section{Funding}

This work was supported by a grant from the National Science Foundation IOS-1146940 (to MEP).

\section{Availability of data and material}

The datasets analyzed during the current study are available from the corresponding author on reasonable request.

\section{Authors' contributions}

SD designed and conducted the experiments and wrote the initial draft of the manuscript. DMB helped with image analysis and oocyte counts. MEP helped with experimental design, data analysis and interpretation and manuscript editing. In addition, MEP obtained funding to support the project. All authors read and approved the final manuscript.

\section{Competing interests}

The authors declare that they have no competing interests.

\section{Consent for publication}

Not applicable.

\section{Ethics approval}

This work was approved by the Syracuse University Institutional Care and Use Committee (IACUC protocol \# 14-006).

\section{Author details}

'Department of Biology, Syracuse University, 107 College Place, Syracuse, NY 13244, USA. ${ }^{2}$ Present Address: University of Michigan Ann Arbor, Ann Arbor, MI, USA.

Received: 12 October 2016 Accepted: 28 November 2016

Published online: 05 December 2016

\section{References}

1. Pepling ME. From primordial germ cell to primordial follicle: mammalian female germ cell development. Genesis. 2006;44:622-32.

2. Borum K. Oogenesis in the mouse. A study of the meiotic prophase. Exp Cell Res. 1961;24:495-507.

3. Pepling ME, Spradling AC. Female mouse germ cells form synchronously dividing cysts. Development. 1998;125:3323-8.

4. Peters $\mathrm{H}$. Migration of gonocytes into the mammalian gonad and their differentiation. Philos Trans R Soc Lond B Biol Sci. 1970;259:91-101.

5. Bullejos M, Koopman P. Germ cells enter meiosis in a rostro-caudal wave during development of the mouse ovary. Mol Reprod Dev. 2004;68:422-8.

6. Pepling ME, Sundman EA, Patterson NL, Gephardt GW, Medico Jr L, Wilson $\mathrm{Kl}$. Differences in oocyte development and estradiol sensitivity among mouse strains. Reproduction. 2010;139:349-57.

7. Hirshfield AN, DeSanti AM. Patterns of ovarian cell proliferation in rats during the embryonic period and the first three weeks postpartum. Biol Reprod. 1995;53:1208-21.

8. McGee EA, Hsueh AJ. Initial and cyclic recruitment of ovarian follicles. Endocr Rev. 2000;21:200-14.

9. Baudat F, Manova K, Yuen JP, Jasin M, Keeney S. Chromosome synapsis defects and sexually dimorphic meiotic progression in mice lacking Spo11. Mol Cell. 2000;6:989-98.

10. Pittman DL, Cobb J, Schimenti KJ, Wilson LA, Cooper DM, Brignull E, Handel MA, Schimenti JC. Meiotic prophase arrest with failure of chromosome synapsis in mice deficient for Dmc1, a germline-specific RecA homolog. Mol Cell. 1998;1:697-705.

11. Paredes A, Garcia-Rudaz C, Kerr B, Tapia V, Dissen GA, Costa ME, Cornea A, Ojeda SR. Loss of synaptonemal complex protein-1, a synaptonemal complex protein, contributes to the initiation of follicular assembly in the developing rat ovary. Endocrinology. 2005;146:5267-77.

12. Ohno S, Smith JB. Role of fetal follicular cells in meiosis of mammalian ooecytes. Cytogenetics. 1964;3:324-33.

13. Byskov AG. Differentiation of mammalian embryonic gonad. Physiol Rev. 1986;66:71-117.

14. Chen Y, Jefferson WN, Newbold RR, Padilla-Banks E, Pepling ME. Estradiol, progesterone, and genistein inhibit oocyte nest breakdown and primordial follicle assembly in the neonatal mouse ovary in vitro and in vivo. Endocrinology. 2007;148:3580-90.

15. Susiarjo M, Hassold TJ, Freeman E, Hunt PA. Bisphenol A exposure in utero disrupts early oogenesis in the mouse. PLoS Genet. 2007;3:e5.

16. Yang MY, Fortune JE. The capacity of primordial follicles in fetal bovine ovaries to initiate growth in vitro develops during mid-gestation and is associated with meiotic arrest of oocytes. Biol Reprod. 2008;78:1153-61.

17. Challoner S. Studies of oogenesis and follicular development in the golden hamster 1. A quantitative study of meiotic prophase in vivo. J Anat. 1974; 117:373-83.

18. Di Carlo AD, Travia G, De Felici M. The meiotic specific synaptonemal complex protein SCP3 is expressed by female and male primordial germ cells of the mouse embryo. Int J Dev Biol. 2000;44:241-4.

19. Wang Y, Teng Z, Li G, Mu X, Wang Z, Feng L, Niu W, Huang K, Xiang X, Wang C, Zhang H, Xia G. Cyclic AMP in oocytes controls meiotic prophase I and primordial folliculogenesis in the perinatal mouse ovary. Development. 2015;142:343-51.

20. Baltus AE, Menke DB, Hu YC, Goodheart ML, Carpenter AE, de Rooij DG, Page DC. In germ cells of mouse embryonic ovaries, the decision to enter meiosis precedes premeiotic DNA replication. Nat Genet. 2006;38:1430-4. 
21. Dokshin GA, Baltus AE, Eppig JJ, Page DC. Oocyte differentiation is genetically dissociable from meiosis in mice. Nat Genet. 2013;45:877-83.

22. Karavan JR, Pepling ME. Effects of estrogenic compounds on neonatal oocyte development. Reprod Toxicol. 2012;34:51-6.

23. Kezele P, Skinner MK. Regulation of ovarian primordial follicle assembly and development by estrogen and progesterone: endocrine model of follicle assembly. Endocrinology. 2003;144:3329-37.

Submit your next manuscript to BioMed Central and we will help you at every step:

- We accept pre-submission inquiries

- Our selector tool helps you to find the most relevant journal

- We provide round the clock customer support

- Convenient online submission

- Thorough peer review

- Inclusion in PubMed and all major indexing services

- Maximum visibility for your research

Submit your manuscript at www.biomedcentral.com/submit 